\title{
Formal Modeling and Verification of Interlocking Systems Featuring Sequential Release
}

\author{
Vu, Linh Hong; Haxthausen, Anne Elisabeth; Peleska, Jan
}

Published in:

Proceedings of the 3rd International Workshop on Formal Techniques for Safety-Critical Systems (FTSCS 2014)

Link to article, DOI:

10.1007/978-3-319-17581-2_15

Publication date:

2015

Document Version

Peer reviewed version

Link back to DTU Orbit

Citation (APA):

Vu, L. H., Haxthausen, A. E., \& Peleska, J. (2015). Formal Modeling and Verification of Interlocking Systems Featuring Sequential Release. In C. Artho, \& P. Csaba Ölveczky (Eds.), Proceedings of the 3rd International Workshop on Formal Techniques for Safety-Critical Systems (FTSCS 2014) (pp. 223-238). Springer.

Communications in Computer and Information Science Vol. 476 https://doi.org/10.1007/978-3-319-17581-2_15

\section{General rights}

Copyright and moral rights for the publications made accessible in the public portal are retained by the authors and/or other copyright owners and it is a condition of accessing publications that users recognise and abide by the legal requirements associated with these rights.

- Users may download and print one copy of any publication from the public portal for the purpose of private study or research.

- You may not further distribute the material or use it for any profit-making activity or commercial gain

- You may freely distribute the URL identifying the publication in the public portal 


\title{
Formal Modeling and Verification of Interlocking Systems Featuring Sequential Release
}

\author{
Linh H. Vu ${ }^{1, \star}$, Anne E. Haxthausen ${ }^{1, \star}$, and Jan Peleska ${ }^{2, \star \star}$ \\ 1 DTU Compute, Technical University of Denmark, Kongens Lyngby, Denmark. \\ \{lvho, aeha\}@dtu.dk \\ 2 Department of Mathematics and Computer Science \\ University of Bremen, Bremen, Germany. \\ jp@informatik. uni-bremen.de
}

\begin{abstract}
In this paper, we present a method and an associated tool suite for formal verification of the new ETCS level 2 based Danish railway interlocking systems. We have made a generic and reconfigurable model of the system behavior and generic high-level safety properties. This model accommodates sequential release - a feature in the new Danish interlocking systems. The generic model and safety properties can be instantiated with interlocking configuration data, resulting in a concrete model in the form of a Kripke structure, and in high-level safety properties expressed as state invariants. Using SMT based bounded model checking (BMC) and inductive reasoning, we are able to verify the properties for model instances corresponding to railway networks of industrial size. Experiments also show that BMC is efficient for finding bugs in the railway interlocking designs.
\end{abstract}

Keywords: Railway interlocking systems · Formal verification $\cdot$ Bounded model checking · Inductive reasoning · RobustRails · Safety-critical systems

\section{Introduction}

An interlocking system is responsible for guiding trains safely through a given railway network. It is a vital part of any railway signaling system and has the highest safety integrity level (SIL4) according to the CENELEC 50128 standard [5]. Conventionally, the development and verification process of interlocking systems is informal and mostly manual, hence time-consuming, costly, and error-prone. Thus, automated verification of interlocking systems is an active research topic, investigated by several research groups, see e.g. [10, $8,23,15,9,14]$. As part of the RobustRailS research project ${ }^{3}$, our work aims at

* The authors' research has been funded by the RobustRailS project granted by the Danish Council for Strategic Research.

** The author's research has been partially funded by ITEA2 project openETCS under grant agreement 11025 .

${ }^{3}$ http://robustrails.man.dtu.dk 
establishing a holistic method supporting the verification of such systems. The method should be formal and facilitate automation in order to provide a better verification process compared to the conventional one. In Denmark, in the period of 2009-2021, new interlocking systems that are compatible with standardized European Train Control System (ETCS) Level 2 [4] will be deployed in the entire country within the context of the Danish Signalling Programme ${ }^{4}$. In the context of the RobustRailS project accompanying the signalling programme on a scientific level, the proposed method will be applied to these new systems.

The main contributions presented in this paper are as follows. (1) We present a formal model of the behavior of ETCS Level 2 compatible interlocking systems. (2) The model accommodates sequential release: this is a method for incrementally releasing route portions that have been traversed by the associated train, with the objective to increase the level of concurrency in route allocation and, consequently, the train throughput. (3) The state space encodings allow for high-level safety properties and state transition relations to be processed in a highly efficient manner by SMT solvers supporting bit vector and integer arithmetics. (4) A verification technique combining induction with bounded model checking (BMC) using novel SMT solvers enables the verification of safety properties for railway network instances of industrial size.

The paper is organized as follows: Section 2 gives a brief introduction to the new Danish route-based interlocking systems. The proposed method is described in Sect. 3. Section 4 presents the formal, generic model in the form of a Kripke structure, while the safety properties are formalized in Sect. 5. Section 6 describes the verification strategy. The experimental results are shown in Sect. 7. Related work and concluding remarks are presented in Sect. 8 and Sect. 9, respectively.

\section{The new Danish Route-based Interlocking Systems}

A railway network in ETCS Level 2 consists of a number of track-side elements of different types ${ }^{5}$ : linear sections, points, marker boards. Figure 1 shows an example layout of a railway network having six linear sections (b10,t10,t12, t14, t20,b14), two points ( $\mathrm{t} 11, \mathrm{t} 13)$, and eight marker boards (mb10..mb21). A linear section is a section with up to two neighbors: one in the up end, and one in the down end ${ }^{6}$, e.g. the linear section t12 in Fig. 1 has t13 and t11 as neighbors at its up end and down end, respectively. A point can have up to three neighbors: one at the stem, one at the plus end, and one at the minus end, e.g. point t11 in Fig. 1 has t10, t12, and t20 as neighbors at its stem, plus, and minus ends, respectively. Linear sections and points are collectively called detection sections, as they are used by interlocking systems to detect the presence of trains in a railway network. A point can be switched between two

\footnotetext{
${ }^{4}$ http://www . bane.dk/signalprogrammet

${ }^{5}$ Here we only show types that are relevant for the work presented in this paper.

${ }^{6}$ In Denmark, up and down denote the directions in which the distance from a reference location is increasing and decreasing, respectively. The location is the same for both up and down, e.g. an end of a line.
} 
positions: PLUS and MINUS. When it is in the PLUS (MINUS) position, traffic can run from its stem to its plus (minus) end and vice versa. A marker board is installed along a section, and it is used as reference location for an intended travel direction that it is facing, e.g. $\mathrm{mb} 13$ in Fig. 1 is installed along section t12, and it is intended for travel direction up. Contrary to legacy systems, in ETCS Level 2, there are no physical signals, but virtual signals associated with marker boards. A virtual signal can be OPEN or CLOSED, respectively, allowing or disallowing traffic to pass the associated marker board. For simplicity, the terms virtual signals, signals, and marker boards are used interchangeably throughout this paper.

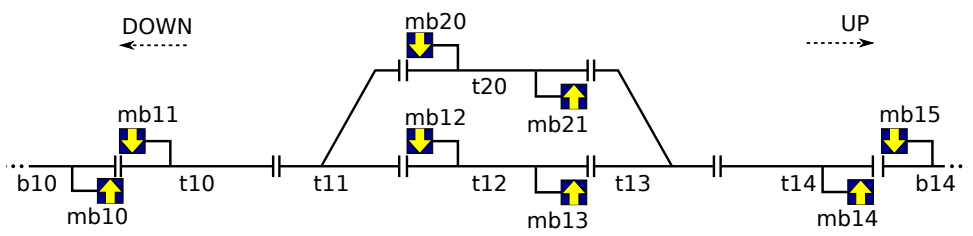

Fig. 1. An example railway network layout

An interlocking system monitors constantly the status of track-side elements, and sets them to appropriate states in order to allow trains traveling safely through the given railway network. The new Danish interlocking systems are route-based. An interlocking table specifies the routes in the given network layout and the conditions for setting these routes. A route is a path from a source signal to a destination signal.

In railway signaling terminology, setting a route denotes the process of allocating the resources - i.e. sections, points, signals - for the route, and then locking it exclusively for only one train when the resources are allocated. The specification of a route and conditions for setting and releasing it include the following information: (a) a list of the detection sections in the route's path, (b) a list of the detection sections which are used as overlaps - buffer space in case trains overshoot the route's path, (c) required positions of points ${ }^{7}$ used by the route, (d) a set of protecting signals used for flank or front protection [19] for the route, and (e) a set of conflicting routes which must not be set while the current route is set.

Table 1 shows an excerpt of an interlocking table for the network shown in Fig. 1. As can be seen, one of the routes has id $1 \mathrm{a}$, goes from mb10 to mb13 via three sections $\mathrm{t} 10, \mathrm{t} 11$ and $\mathrm{t} 12$, and has no overlap. It requires point $\mathrm{t} 11$ (on its path) to be in PLUS position and point t13 (outside its path) to be in MINUS position (as a protecting point). The route has mb11, mb12 and mb20 as

\footnotetext{
7 This includes points in the path and overlaps, and points used for flank and front protection. For detail about flank and front protection, see [19].
} 
protecting signals, and it is in conflict with routes $1 \mathrm{~b}, 2 \mathrm{a}, 2 \mathrm{~b}, 3,4,5 \mathrm{a}, 5 \mathrm{~b}, 6 \mathrm{~b}$, and 7 .

Table 1. Excerpt of the interlocking table for the network layout in Fig. 1. The overlaps column is omitted as it is empty for all of the routes. (p means PLUS, m means MINUS.)

\begin{tabular}{|l|l|l|l|l|l|l|}
\hline id & source & dest. & points & signals & path & conflicts \\
\hline $1 \mathrm{a}$ & $\mathrm{mb} 10$ & $\mathrm{mb} 13$ & $\mathrm{t} 11: \mathrm{p} ; \mathrm{t} 13: \mathrm{m}$ & $\mathrm{mb} 11 ; \mathrm{mb} 12 ; \mathrm{mb} 20$ & $\mathrm{t} 10 ; \mathrm{t} 11 ; \mathrm{t} 12$ & $1 \mathrm{~b} ; 2 \mathrm{a} ; 2 \mathrm{~b} ; 3 ; 4 ; 5 \mathrm{a} ; 5 \mathrm{~b} ; 6 \mathrm{~b} ; 7$ \\
.. & $\ldots$ & $\ldots$ & $\ldots$ & $\ldots$ & $\ldots$ & $\ldots$ \\
7 & $\mathrm{mb} 20$ & $\mathrm{mb} 11$ & $\mathrm{t} 11: \mathrm{m}$ & $\mathrm{mb} 10 ; \mathrm{mb} 12$ & $\mathrm{t} 11 ; \mathrm{t} 10$ & $1 \mathrm{a} ; 1 \mathrm{~b} ; 2 \mathrm{a} ; 2 \mathrm{~b} ; 3 ; 5 \mathrm{~b} ; 6 \mathrm{a}$ \\
\hline
\end{tabular}

Interlocking Principles. In order to prevent collision and derailment of trains, traditional route-based interlocking systems employ a basic principle: a route is locked exclusively for use of one train at a time. This is obtained by following a strict procedure for setting and releasing routes based on information in their interlocking tables. As an example, let us consider the following procedure for route 1a specified in Table 1:

(0) Initially the route is free.

(1) When a request for setting the route is received by the interlocking system, the route is marked as requested.

(2) The interlocking system checks the status of different track-side elements in the system to figure out whether it can start allocating resources for route 1a, e.g. sections t10, t11 and t12 must be vacant, and conflicting routes must not be allocated or locked. If so, the interlocking commands points and signals to their required positions according to the route's specification, e.g. it commands the point $t 11$ to switch to PLUS, t13 to switch to MINUS, and the protecting signals mb11, mb12 and mb20 to change to CLOSED.

(3) The interlocking system constantly monitors the status of the trackside elements. When the signals and points have changed their states as commanded in step (2), the route is locked and its source signal mb10 is set to OPEN, allowing a train to enter the route.

(4) When the locked route is used, i.e. a train enters it, the source signal mb10 is set to CLOSED preventing other trains from entering.

(5) The route is released (set back to free) when the train has finished using it, i.e. the train has passed mb13, or the train has come to standstill in front of mb13.

Sequential Release. The new Danish interlocking systems employ sequential release (also known as sectional release) [19]. This feature results in two major changes:

(a) With sequential release, the interlocking can release an element in a locked route as soon as the train has passed it, instead of waiting until the train has finished using the route and then releasing the route as a whole. Consequently, the capacity increases. 
(b) As a direct result of (a), a route may be allocated (in step (2) above) while some of its conflicting routes are still in use by trains, instead of waiting for all of its conflicting routes to be released as in traditional route-based interlocking systems. For example, when a train has passed section t11 while going along route $1 \mathrm{a}$, t11 will be released and then route 7 going in the opposite direction (see Table 1) can be allocated (assuming that other conditions for this are fulfilled).

\section{Verification Method}

The verification process is shown in Fig. 2.The verification process begins with the configuration data of an interlocking system, consisting of a network layout and an interlocking table. The configuration data are described in a domainspecific language [22] (DSL) having an XML representation ${ }^{8}$. After being parsed into an internal representation, a static checker verifies whether the configuration data is statically well-formed according to the static semantics of the DSL. As an option the user may not provide an interlocking table, but instead use an interlocking table generator (ITG) to get a table created automatically. Instantiating a generic model of the dynamic behavior of the Danish interlocking systems with the well-formed configuration data results in a model instance in the form of a Kripke structure. Similarly, the concrete safety-properties expressed as state invariants are also generated from the generic safety- properties. The model instance is then checked against the concrete properties using a combination of BMC and inductive reasoning. If the model instance does not satisfy the properties, counter-examples will be generated. An interface for visualizing the counter-examples at the DSL level is under development.

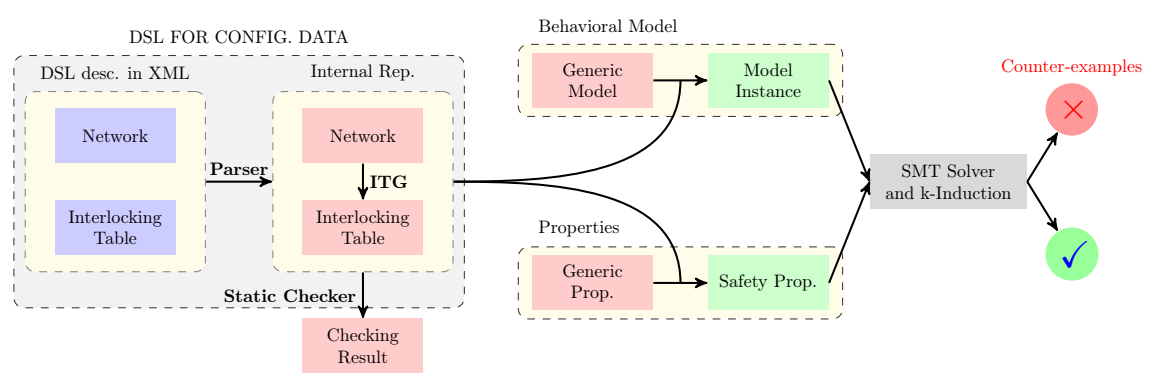

Fig. 2. Verification process

The tool-chain associated with the method has been implemented using the RT-Tester tool-box $[17,21]$. The bounded model checker in RT-Tester uses the SONOLAR SMT solver [18] to compute counter-examples for induction and

\footnotetext{
${ }^{8}$ A graphical representation and editor is currently under development.
} 
base cases. RT-Tester has been selected because (1) it is an integrated modelbased testing and BMC tool, and (2) its SMT solver also supports floating point arithmetic. The first property is crucial for us, because our objective is to complement the model verification with $\mathrm{HW} / \mathrm{SW}$ integration tests. The second capability is vital, because we also plan to extend the model by real-time aspects, such as train velocity and braking curves.

\section{Kripke Structure Encodings of Interlocking Systems}

The dynamic behavior of an interlocking system is formalized as a Kripke structure $K=\left(S, s_{0}, R, L, A P\right)$ with state space $S$, initial state $s_{0} \in S$, transition relation $R \subseteq S \times S$, and labeling function $L: S \rightarrow 2^{A P}$, where $A P$ is the set of atomic propositions and $2^{A P}$ is the power set of $A P$. The labeling function $L$ maps a state $s$ to the set $L(s)$ of atomic propositions that hold in $s$. Due to the limited space of this paper and the complexity of the Kripke encodings, in the following subsections, we only outline how the state space $S$ and the transition relation $R$ of a Kripke structure are encoded.

\subsection{State Space}

In order to encode the states of an interlocking system, a finite set $V=$ $\left\{v_{0}, \ldots, v_{n}\right\}$ of variables is defined to represent the current status of different components in the system such as a track element or a route. Each variable $v \in V$ has an associated finite domain $D_{v} \subset \mathbb{N}_{0}$. The state space is the set of all valuation functions $s: V \rightarrow \bigcup_{v \in V} D_{v}$ for which $s(v) \in D_{v}$ for all $v \in V$. The initial state $s_{0}$ is the (safe) state in which all detection sections are vacant, all signals are closed, all routes are free, and there are no trains in the network. In our encodings, $s_{0}$ is the state in which all variables are evaluated to 0 . For readability, sometimes we use named constants instead of their corresponding integral values in the subsequent paragraphs.

Vacancy Status. The vacancy status of a section in a given travel direction is encoded using the three least significant bits HTO of a non-negative integer variable as shown in Fig. 3. For example, the variable $l$. U2D records the vacancy status of a linear section $l$ in the direction from its up end to its down end. The value 1 of the bits H, T, $\mathrm{O}$ indicate: (H) the head of the train is within the section, (T) the tail of the train is within the section, and ( 0 ) the section is occupied, respectively. This encoding offers two advantages: $(a)$ the encoding can cover the case where a train occupies more than one detection section (e.g., when it is crossing the joint between two sections), and (b) the safety properties can be expressed efficiently using arithmetic operations on integer variables as shown in Sect. 5.

Lockable Elements. In order to accommodate sequential release into our model, we consider a linear or point section as a lockable element. The status of a lockable 


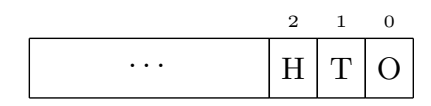

Fig. 3. A variable recording occupancy status of a detection section

element $e$ is encoded by two variables: (1) e.MODE - indicating the mode of the element, and (2) e.PREV - this variable is set to 1 when the previous section in the same route has been released, otherwise e.PREV $=0$. An element can be in one of the following modes: FREE (the element is not exclusively locked by a route, or used by any train), EXLCK (the element is exclusively locked for a route), or USED (the element has been used, i.e., occupied, by a train after it was exclusively locked for a route).

Point Positions. The position of a point $p$ is encoded by two variables: (1) p.POS - the actual position of the point, and (2) p.CMD - the point position commanded by the interlocking. The value of p.POS can be one of the following ${ }^{9}$ : PLUS(0), MINUS(1), or INTERMEDIATE(2) (the position where the point is switching from one side to the other). The value of $p . C M D$ can only be PLUS or MINUS (as the interlocking cannot command a point to switch to the INTERMEDIATE position).

Signal Aspects. The aspect of a signal $s$ is encoded by two variables: (1) s.ACT - the actual aspect of the virtual signal, its value can be OPEN or CLOSED, and (2) s.CMD - the aspect as commanded by the interlocking, the possible values of this variable have the same meaning as the ones of s.ACT. The s.ACT variable represents the aspect of the signal as "seen" by the train, while $s . C M D$ is the aspect of the signal as seen by the interlocking. The values of these two variables may be different because of the delay in the communication between the interlocking system and the trains.

Routes. For each route $r$, a variable r.MODE is used to encode the current mode of that route. A route can be in one of the following modes: FREE, MARKED, ALLOCATING, LOCKED, or USED.

\subsection{Transition Relation}

The transition relation $R \subseteq S \times S$ can be represented symbolically by a predicate $\Phi$ with free variables in $V \cup V^{\prime}$, where $V^{\prime}=\left\{v^{\prime} \mid v \in V\right\}$ is the set of next-state variables. A pair of states $\left(s, s^{\prime}\right) \in R$, if and only if $\Phi$ evaluates to true when replacing every $v \in V$ occurring in $\Phi$ with $s(v)$ and every $v^{\prime} \in V^{\prime}$ occurring in $\Phi$ with $s^{\prime}(v)$. In order to specify $\Phi$, we divide the transitions in an interlocking system into four types as in the following, each type is represented collectively in a predicate with free variables in $V \cup V^{\prime}$.

\footnotetext{
9 The notation name(integer-value) means that name is the name of constant having the value integer-value.
} 
(0) route dispatching transitions represented collectively by the predicate $\Phi_{d}$;

(1) interlocking transitions - e.g., setting mode of a route - represented by the predicate $\Phi_{\iota}$

(2) track element transitions - e.g., switching a point or a signal - represented by the predicate $\Phi_{\epsilon}$; and

(3) train movement transitions represented by the predicate $\Phi_{\tau}$.

Transitions of type (0) are not prioritized, i.e., they can be chosen whenever they are enabled, independently from other transitions. On the other hand, transitions of types (1), (2), and (3) are prioritized in the descending order that they appear in the list, i.e., transitions of type (1) has the highest priority and transitions of type (3) has the lowest. Whenever two transitions of different priorities are both enabled, the one with higher priority will be chosen. Transitions with the same priority are chosen non-deterministically if they are enabled at the same time. This priority of transitions is based on the intuition that in practice, the events in the interlocking control logic occur at significantly higher speed than the ones occurring in a track element. An analogous argument applies to events related to track elements and others related to train movements. With these types of transitions, the transition relation of an interlocking system can be specified as in the following

$$
\Phi \equiv \Phi_{d} \vee \operatorname{ITE}\left(\iota, \Phi_{\iota}, \operatorname{ITE}\left(\epsilon, \Phi_{\epsilon}, \Phi_{\tau}\right)\right)
$$

where $\operatorname{ITE}(c, i, e)$ is the if-then-else function: if $c$ holds then the value of the function is $i$, otherwise it is $e ; \iota$ expresses whether an interlocking transition is enabled; and $\epsilon$ expresses whether a track element transition is enabled. The route dispatching transition relation $\Phi_{d}$ is put outside of the ITE function in (1) in order to allow the routes to be dispatched arbitrarily. If route dispatching transitions were given the same or higher priority as the one of interlocking control logic transitions, all routes which could be dispatched would have to be dispatched before track elements or trains could make any transition. On the other hand, if route dispatching were given lower priority than interlocking control logic transitions, then a route could not be dispatched if another route is processed by the interlocking.

Route Dispatching. A route can be dispatched arbitrarily whenever its mode is FREE. This means that multiple routes can be dispatched at the same time.

Life-cycle of a Route. Figure 4 shows the "life-cycle" of a route, i.e., its different modes and the transitions from one mode to another. This "life-cycle" reflects the procedure for setting and sequentially releasing a route as described in Sect. 2. The transitions labeled (1), (2), (3), (4), and (6) in Fig. 4 correspond to items $(1)-(5)$ in the procedure presented in Sect. 2 for setting and releasing a route. Transition (5) models the sequential release that can take place while the route stays in USED mode: as the train moves along the route, its elements are released sequentially as soon as the train has passed them. Transition (2) is adapted to sequential release: allocation is now also allowed when a conflicting route is in the USED mode, as long as elements shared with the given route have been sequentially released. 


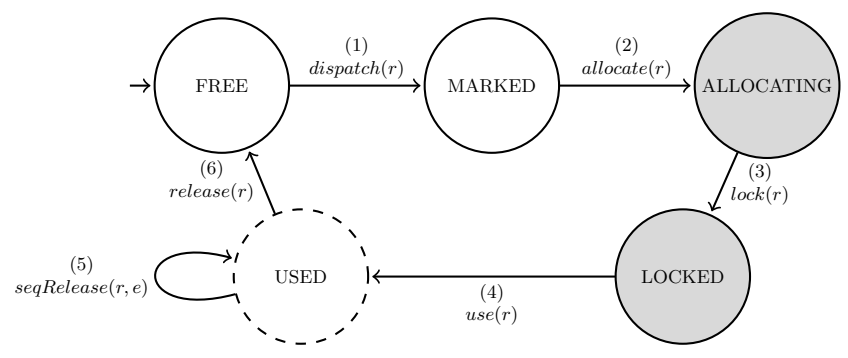

Fig. 4. A life-cycle of a route

Life-cycle of a Lockable Element. Figure 5 depicts the "life-cycle" of a lockable element within the network controlled by the interlocking system. Each node in the diagram is labeled with information about the status of the element $e$ : (a) whether the element is vacant, (b) its current mode, and (c) the value of the PREV variable indicating whether the previous element prev $(r, e)$ of $e$ in the route $r$ has been released. An element $e$ is initially in a state in which it is vacant, in FREE mode, and its PREV variable is 0. (1) When the interlocking system is allocating a route $r$ that uses $e$, it sets the mode of the element to EXLCK, meaning that the element is locked exclusively for $r$. (2) The element becomes occupied, i.e., not vacant, as a train enters. (3) After that, e's mode is set to USED. (4) When the train leaves the previous element $\operatorname{prev}(r, e)$ of $e$ in the route $r, \operatorname{prev}(r, e)$ is released, and it informs $e$ by setting the variable e.PREV to 1. (5) When the train leaves $e$, the latter becomes vacant again, (6) $e$ is released and the next element $n \operatorname{ext}(r, e)$ in the same route is informed by setting next $(r, e)$.PREV to 1 .

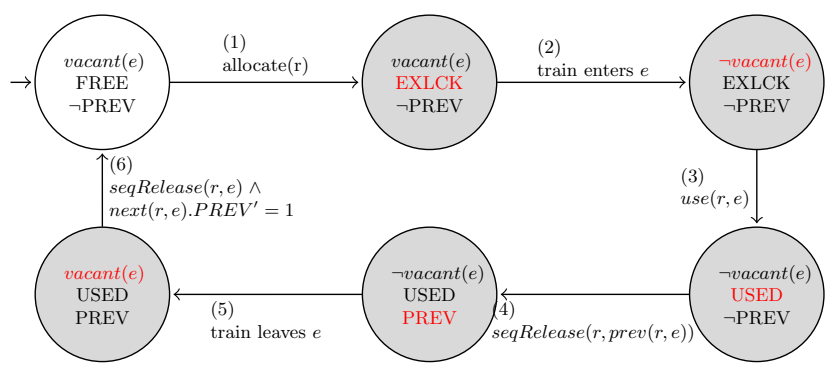

Fig. 5. "Life-cycle" of a lockable element $e$. vacant $(e)$ is a formula over variables encoding e's vacancy status shown in Sect. 4.1.

Switching Points. A point $p$ can be switched if it is requested to be switched to a position p.CMD that is different from its current position p.POS. The point switching process occurs in two steps: 
(1) the point moves from its current position to the intermediate position, i.e., $p . P O S \neq$ p.CMD $\wedge$ p.POS $\neq$ INTERMEDIATE $\wedge$ p.POS $=$ INTERMEDIATE,

(2) the point is switched from the intermediate position to the requested position, i.e., $p . P O S=I N T E R M E D I A T E \wedge p . P O S^{\prime}=p . C M D$.

Switching Signals. Whenever the actual aspect $s . A C T$ of a signal $s$ differs from the commanded aspect s.CMD, the actual aspect of the signal is set to the commanded aspect, i.e., $s . A C T \neq s . C M D \wedge s . A C T^{\prime}=$ s. $C M D$.

Train Movements. Trains are not explicitly specified in our model, in the sense that there are no explicit train objects. Instead, train movements and other aspects are implicitly modeled via the occupancy status of train detection sections, inspired by the "rubber-band" model described in [1]. This implicit model is advantageous when compared to the explicit one, because it models arbitrary numbers of trains of arbitrary length. In the implicit model of train movements, train length - in terms of numbers of sections that a train occupies - may vary as trains move. This variation reflects the actual view of interlocking systems of the train length: although trains have fixed geometric length, their length - in terms of the number of sections that they occupy - as seen by the interlocking systems is not fixed.

\section{$5 \quad$ High-level Safety Properties}

Interlocking systems must at least guarantee the high-level safety properties of non-collision and non-derailment. These properties can be expressed as state invariants over the vacancy status variables of linear and point sections in the given network. Basically, an interlocking system is safe if no hazardous situations occur on any linear or point section at any time. Thus, the high- level safety properties can be expressed formally by the following state invariant

$$
\phi=\neg\left(\bigvee_{l: \text { Linear }} \text { Hazard }_{l} \vee \bigvee_{p: \text { Point }} \text { Hazard }_{p}\right)
$$

where $\operatorname{Hazard}_{l}$ and Hazard $p$ specify conditions for hazards to occur on a linear section $l$ and a point $p$, respectively. These propositions are disjunctions of sub-propositions expressing hazards of different types on a section such as: (a) head-to-head collision, (b) trains following each other collision on a section, or (c) derailment on a point. Some examples of sub-propositions are given in the subsequent paragraphs.

Head-to-head collision on a linear section. A head-to-head collision occurs on a linear section $l$, when two trains running in opposite directions meet. This situation is expressed by the following formula where l.D2U (l.U2D) is the variable encoding the vacancy status of the section in the travel direction from down (up) to up (down).

$$
\text { l.D2U } * l . U 2 D>0
$$


As $l . D 2 U * l . U 2 D>0$ iff $l . D 2 U>0$ and $l . U 2 D>0$, the formula expresses that the section is occupied in both down-to-up $($ l.D2U $>0)$ and up-to-down $(l . U 2 D>0)$ directions. Collisions of type (b) are formulated in the similar way.

Derailment on a point. A derailment occurs when a train traverses a point $p$ which is not locked in the correct position for the travel direction of the train. This situation is expressed by the following formula where p.POS is the point's actual position, $p . S 2 P M, p . P 2 S$, and $p . M 2 S$ are variables encoding the vacancy status of the point in the travel direction entering the point from its stem, plus, or minus ends, respectively, \& and $\gg$ are bit-wise and and arithmetic bit shift right operators, respectively.

$$
p . P O S * p . P 2 S+(1-(p . P O S \& 1)) * p . M 2 S+(p . P O S \gg 1) * p . S 2 P M>0
$$

Formula (4) captures the following cases: (a) a train is entering a point from its plus end $(p . P 2 S>0)$ while the point is in not in the plus position $(p \cdot P O S>0)$, (b) a train is entering a point from its minus end $(p . M 2 S>0)$ while the point is not in the minus position $(1-(p . P O S \& 1)>0)$, and (c) a train is entering a point from its stem end $(p . S 2 P M>0)$ while the point is in the intermediate position $((p . P O S \gg 1)>0)$.

\section{Verification of Safety Properties}

When a model $K$ (see Sect. 4) and a proposition $\phi$ expressing high-level safety properties (see Sect. 5) have been generated, the next task according to our method is to prove the absence of hazardous situations, i.e., to prove that $\phi$ holds in all reachable states of $K$. This is written $K=G(\phi)$ where $G$ is Globally temporal operator in Linear Temporal Logic (LTL). The following subsections describe our approach for verifying this.

\subsection{Verification Strategy}

We employ a strategy combining BMC and $k$-induction techniques similar to the one in [13]. The verification procedure is performed in two steps: (i) base case: prove that $\phi$ holds for $k>0$ consecutive states ${ }^{10}$, starting from the initial state $s_{0}$, and (ii) induction case: prove that if $\phi$ holds for $k>0$ consecutive states, starting from an arbitrary state $s_{n}$, then $\phi$ will also hold in the $(k+1)^{t h}$ state. Both the base case and the induction case are transformed to problems of finding counter-examples for their negated formulas using an SMT solver. If no counter-examples are found, then the cases have been proved.

${ }^{10}$ Two states are consecutive, if there is a transition from the first to the second according to the model $K$. 


\subsection{Invariant Strengthening}

As pointed out in [3], when $\phi$ is not strong enough to be inductive, counterexamples are found for the induction case. These counter- examples are often spurious, i.e., they start from an unreachable state and do not correspond to any actual run of the system. In order to make $\phi$ inductive, it is strengthened with an extra invariant $\psi$, i.e., one should prove $\phi \wedge \psi$ instead of $\phi . \psi$ is called the strengthening invariant, which eliminates the spurious counter-examples. An example of such strengthening properties is given in the following.

Train Integrity. Some states of the variables expressing the train occupancy status of the track sections (see Sect. 4) are not feasible as they correspond to situations that are not physically possible. An example of an infeasible state is one in which the variables express that a section $s$ is occupied in one direction by a train without the head being on the section, but the next section in that travel direction is unoccupied.

The train integrity conditions can be formalized as a conjunction of formulas over the track vacancy variables. For each travel direction ( $u p$ and down), there is a formula for each section $s$ that has a next section in the given travel direction. The pattern of such a formula depends on the other sections the current section is connected to in the given travel direction. For instance, for travel direction up and a linear section $s$ that has a linear section $s^{\prime}$ as neighbor in travel direction $u p$, the formula will take the following form:

$$
(s . D 2 U \& 0 b 101)=0 b 001 \Longleftrightarrow\left(s^{\prime} . D 2 U \& 0 b 011\right)=0 b 001
$$

where \& is the bit-wise and operator. This formula expresses that section $s$ is occupied by a train in direction up (the $O$ bit of s.D2U is 1) without the head being on the section (the $H$ bit of $s . D 2 U$ is 0 ), if and only if section $s^{\prime}$ is occupied by a train in direction $u p$ (the $O$ bit of $s^{\prime}$.D2U is 1 ) without a tail being on the section (the $T$ bit of $s^{\prime}$.D2U is 0 ). Formula (5) shows the expressiveness of our state encodings allowing properties to be efficiently formulated in compact formulas.

\section{Experiments}

We have used the tool-chain to verify the safety properties for model instances of a number of railway networks, ranging from a trivial tiny toy network to a large station (Køge) extracted from the early deployment line of the new Danish signalling systems.

In our first trials of verifying the models, we used simple induction (kinduction with $k=1$ ), but we got spurious counter-examples. To avoid that we tried to increase $k$ and strengthen the invariant to be verified. It turned out that the verification time increased significantly as $k$ increased, making it impossible to verify even the small networks. However, we were able to derive strengthening properties $\psi$ (see Sect. 6) for which the verification could be 
Table 2. Verification results for different networks using simple induction $(k=1)$. Toy, cross, and mini are made-up trivial networks, while Gadstrup-Havdrup (Gt-Hd) and Køge are extracted from the early deployment line in the Danish Signalling Programme. (BR: branching ratio)

\begin{tabular}{|l|r|r|r|r|r|r|r|r|}
\hline Case & Linears & Points & Signals & Routes & BR & Vars & Time(sec) & Memory(MB) \\
\hline Toy & 6 & 1 & 6 & 4 & 0.17 & 47 & 2 & 63 \\
Cross & 8 & 2 & 8 & 10 & 0.25 & 72 & 9 & 137 \\
Mini & 6 & 2 & 8 & 12 & 0.33 & 66 & 11 & 128 \\
Gt-Hd & 21 & 5 & 24 & 33 & 0.24 & 200 & 146 & 626 \\
Køge & 57 & 23 & 60 & 73 & 0.40 & 582 & 3868 & 4457 \\
\hline
\end{tabular}

done just using simple induction. (Not for all applications this is possible, see, e.g., [13]). Table 2 shows the results of the final verification. Each row of the table lists the size of a network in terms of the number of linear sections, points, signals, and routes in the configuration, and the number of generated variables in the corresponding model instance. The two last columns show the approximate accumulated verification time and memory usage. All experiments have been performed on Intel(R) Core(TM) i7-3520M CPU @ 2.90GHz, 8GB RAM, Ubuntu 14.04 LTS, Linux 3.14.1-031401-generic x86_64 kernel.

The branching ratio of a network (BR in Table 2) is defined as the ratio of the number of points to the number of linear sections in that network. The larger the branching ratio is, the more complex the corresponding network is in terms of branching. The size of the formula $\Phi$ specifying the transition relation as well as the size of the formulas $\phi$ and $\psi$ specifying the state invariants grow as the size of the network grows. Our experiments show that the formulas grow much more when the network's branching ratio also increases, than when the branching ratio is nearly the same (as it is, e.g., the case when chaining multiple simple stations). This is due to the fact that the interdependency between variables in the model also increases when BR increases.

We also injected errors into models. Counter examples for these were normally found in relatively short time. This appears to be a general trend when dealing with interlocking systems [16]. In a few cases, it took long time to find counter examples. Such examples usually represent very subtle errors in the model or the configuration data, which may be easily overlooked by inspection.

\section{Related Work}

In recent years, the railway domain has become one of the most promising application domains of formal methods. Several research groups have investigated how formal methods would help efficiently producing more robust railway control systems. An overview of recent trends can be found in [7], and recommendations and best-practices for efficient development and verification of safe railway control systems are summarized in [12]. Re-configurable systems and automated verification are among these recommendations that we have followed. 
Model checking is a promising technique for verifying safety properties of interlocking systems thanks to its capability to be fully automated. Unfortunately, due to the state explosion problem, the technique is only able to verify applications of small size [8]. Several techniques have been proposed in order to push the applicability bounds toward industrial size. Winter et al. suggest using ordering strategies optimized for interlocking models [23]. A number of high-level abstractions for reducing the complexity of interlocking models are presented in [15]. In [6], Fantechi et al. suggest a distributed interlocking model whose verification can be divided into small tasks and verified in parallel. SATbased model checking and slicing technique are used in [16]. In order to remedy the problem with state space explosion in the global model checking approach, we have recently for some other applications $[13,14]$ used BMC instead. In the current work, a combination of SMT-based BMC with inductive reasoning allowed us to verify safety properties without having to explore the whole state space, hence we were able to push the bounds even further to handle larger networks of industrial size. As an alternative to the model checking approach, theorem proving based techniques have also shown success in the railway domain, see, e.g., $[2,11]$, but are less automated.

Although sequential release has been used in some interlocking systems, we have not found any published formal models of interlocking systems that integrate this feature. In [20], the conditions for elements to be unlocked and reused in sequential releases are pre-computed and specified in the interlocking tables. In our approach, sequential release is integrated into the behavioral model rather than into the configuration data. This reduces the complexity of the configuration data and makes interlocking configuration data relatively independent from the chosen interlocking approaches.

\section{Conclusion and Future Work}

This paper presented a fully automated, formal method and an associated tool suite for verifying the forthcoming new ETCS Level 2 based Danish railway interlocking systems featuring sequential release. A formal model for these systems was outlined. A novelty in our contribution is that the system is part of an ETCS Level 2 based signalling system in which there are no physical signals along the tracks; instead, movement authorities are communicated via onboard computers. By introducing the concept of virtual signals, we have been able to handle the assignment of movement authorities in a way that is very similar to the situations where conventional signals are used. Another novelty is that the formal model features sequential release. As a consequence, the model is more complex than those supporting route-based release only, because additional variables and transitions are required. Therefore the verification becomes more challenging. In spite of this difficulty, using a combination of SMT-based BMC and inductive reasoning, we were able to successfully verify safety properties for systems controlling large networks of realistic size. This was enabled by encodings of the state space, the transition relation, and of the safety properties 
that can be efficiently evaluated by SMT solvers supporting bit vector and integer arithmetics.

In order to compare our verification approach to the approaches that use BDD-based symbolic model checking, a translation from our model to NuSMV - a well-known BDD-based symbolic model checker - is currently in progress. For future work, we will benchmark how sequential release affects the complexity, and hence verification challenges, of interlocking models. Furthermore, we will investigate advanced techniques for automating the process of discovering strengthening invariants, or reducing the size of the networks that need to be modeled. For the current model there are potential overlaps between the strengthening invariants, which should be eliminated in order to reduce the size of the formula to be solved by the SMT solver.

Acknowledgments. The authors would like to thank Ross Edwin Gammon and Nikhil Mohan Pande from Banedanmark (Railnet Denmark) and Jan Bertelsen from Thales for helping us with their expertise about Danish interlocking systems and always being helpful when we had questions; and Dr.-Ing. Uwe Schulze and Florian Lapschies from University of Bremen for their help with the implementation in the RT-Tester tool-chain.

\section{References}

1. Morten Aanæs and Hoang Phuong Thai. Modelling and verification of relay interlocking systems. Master's thesis, Technical University of Denmark, DTU Informatics, 2012. Series: IMM-MSC-2012-14.

2. Salimeh Behnia, Amel Mammar, Jean-Marc Mota, Nicolas Breton, Paul Caspi, and Pascal Raymond. Industrialising a Proof-based Verification Approach of Computerised Interlocking Systems. In J. Allan, E. Arias, C.A. Brebbia, C. Goodman, A.F. Rumsey, G. Sciutto, and N. Tomii, editors, Eleventh International Conference on Computer System Design and Operation in the Railway and Other Transit Systems (COMPRAIL08). WIT Press, 2008.

3. Leonardo Mendonça de Moura, Harald Rueß, and Maria Sorea. Bounded model checking and induction: From refutation to verification. In Warren A. Hunt Jr. and Fabio Somenzi, editors, Computer Aided Verification, 15th International Conference, CAV 2003, Boulder, CO, USA, July 8-12, 2003, Proceedings, volume 2725 of Lecture Notes in Computer Science, pages 14-26. Springer, 2003.

4. ERTMS. Annex A for ETCS Baseline 3 and GSM-R Baseline 0, April 2012.

5. CENELEC European Committee for Electrotechnical Standardization. EN 50128:2011 - Railway applications - Communications, signalling and processing systems - Software for railway control and protection systems. 2011.

6. Alessandro Fantechi. Distributing the Challenge of Model Checking Interlocking Control Tables. In Tiziana Margaria and Bernhard Steffen, editors, Leveraging Applications of Formal Methods, Verification and Validation. Applications and Case Studies, volume 7610 of Lecture Notes in Computer Science, pages 276-289. Springer, 2012.

7. Alessandro Fantechi. Twenty-Five Years of Formal Methods and Railways: What Next? In Steve Counsell and Manuel Núñez, editors, Software Engineering and 
Formal Methods, volume 8368 of Lecture Notes in Computer Science, pages 167183. Springer, 2014.

8. Alessio Ferrari, Gianluca Magnani, Daniele Grasso, and Alessandro Fantechi. Model Checking Interlocking Control Tables. In Eckehard Schnieder and Géza Tarnai, editors, FORMS/FORMAT 2010 - Formal Methods for Automation and Safety in Railway and Automotive Systems, pages 107-115. Springer, 2010.

9. Helle Hvid Hansen, Jeroen Ketema, Bas Luttik, Mohammad Reza Mousavi, Jaco van de Pol, and Osmar Marchi dos Santos. Automated Verification of Executable UML Models. In Bernhard K. Aichernig, Frank S. de Boer, and Marcello M. Bonsangue, editors, FMCO, volume 6957 of Lecture Notes in Computer Science, pages 225-250. Springer, 2010.

10. Anne E. Haxthausen, Marie Le Bliguet, and Andreas A. Kjær. Modelling and Verification of Relay Interlocking Systems. In Christine Choppy and Oleg Sokolsky, editors, 15th Monterey Workshop: Foundations of Computer Software, Future Trends and Techniques for Development, number 6028 in Lecture Notes in Computer Science, pages 141-153. Springer, 2010. Invited paper.

11. Anne E. Haxthausen and Jan Peleska. Formal Development and Verification of a Distributed Railway Control Systems. In IEEE Transactions on Software Engineering, volume 26, pages 687-701. IEEE, 2000.

12. Anne E. Haxthausen and Jan Peleska. Efficient Development and Verification of Safe Railway Control Software. In Railways: Types, Design and Safety Issues, pages 127-148. Nova Science Publishers, Inc., 2013.

13. Anne E. Haxthausen, Jan Peleska, and Sebastian Kinder. A Formal Approach for the Construction and Verification of Railway Control Systems. In Formal Aspects of Computing, volume 23, pages 191-219. Springer, 2011.

14. Anne E. Haxthausen, Jan Peleska, and Ralf Pinger. Applied Bounded Model Checking for Interlocking System Designs. In Steve Counsell and Manuel Núñez, editors, Software Engineering and Formal Methods, volume 8368 of Lecture Notes in Computer Science, pages 205-220. Springer, 2014.

15. Philip James, Faron Möller, Hoang Nga Nguyen, Markus Roggenbach, Steve Schneider, Helen Treharne, Matthew Trumble, and David Williams. Verification of Scheme Plans Using CSP\|B. In Steve Counsell and Manuel Núñez, editors, Software Engineering and Formal Methods, volume 8368 of Lecture Notes in Computer Science, pages 189-204. Springer, 2014.

16. Phillip James and Markus Roggenbach. Automatically Verifying Railway Interlockings Using SAT-based Model Checking. In Electronic Communications of the EASST, volume 35. EASST, 2011.

17. Jan Peleska. Industrial-Strength Model-Based Testing - State of the Art and Current Challenges. In Alexander K. Petrenko and Holger Schlingloff, editors, Proceedings 8th Workshop on Model-Based Testing, Rome, Italy, volume 111 of Electronic Proceedings in Theoretical Computer Science, pages 3-28. Open Publishing Association, 2013.

18. Jan Peleska, Elena Vorobev, and Florian Lapschies. Automated Test Case Generation with SMT-Solving and Abstract Interpretation. In Mihaela Gheorghiu Bobaru et al., editor, NASA Formal Methods, volume 6617 of Lecture Notes in Computer Science, pages 298-312. Springer, 2011.

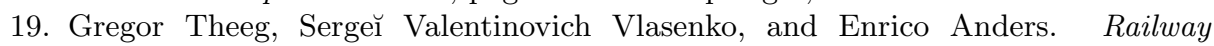
Signalling $\&$ Interlocking: International Compendium. Eurailpress, 2009.

20. David Tombs, Neil Robinson, and George Nikandros. Signalling Control Table Generation and Verification. In CORE 2002: Cost Efficient Railways through 
Engineering, page 415. Railway Technical Society of Australasia/Rail Track Association of Australia, 2002.

21. Verified Systems International GmbH. RT-Tester Model-Based Test Case and Test Data Generator - RTT-MBT - User Manual, 2013.

22. Linh $\mathrm{H} . \mathrm{Vu}$, Anne E. Haxthausen, and Jan Peleska. A Domain-Specific Language for Railway Interlocking Systems. In Eckehard Schnieder and Géza Tarnai, editors, FORMS/FORMAT 2014 - 10th Symposium on Formal Methods for Automation and Safety in Railway and Automotive Systems, pages 200-209. Institute for Traffic Safety and Automation Engineering, Technische Universität Braunschweig, 2014.

23. Kirsten Winter. Optimising Ordering Strategies for Symbolic Model Checking of Railway Interlockings. In Tiziana Margaria and Bernhard Steffen, editors, Leveraging Applications of Formal Methods, Verification and Validation. Applications and Case Studies, volume 7610 of Lecture Notes in Computer Science, pages 246-260. Springer, 2012. 